\title{
Correction to: Integrating Additive Manufacturing into a Virtual Industry 4.0 Factory
}

Mohammad Azarian, Hao Yu, and Wei Deng Solvang

\section{Correction to:}

Chapter "Integrating Additive Manufacturing into a Virtual Industry 4.0 Factory" in: Y. Wang et al. (Eds.):

Advanced Manufacturing and Automation X, LNEE 737, https://doi.org/10.1007/978-981-33-6318-2_73

In the original version of the book, the following correction has been incorporated: The author's affiliation has been linked with the author's name "Mohammad Azarian" in the XML of Chapter 73. The book and the chapter have been updated with the change. 